\title{
Migraine and tension headache comorbidity with hypothyroidism in Egypt
}

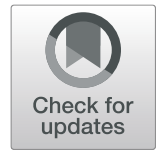

\author{
Ali A. Abou Elmaaty ${ }^{1 *}$, Mohamed E. Flifel ${ }^{2}$, Tamer Belal ${ }^{2}$ and Carmen A. Zarad ${ }^{3}$
}

\begin{abstract}
Background: Migraine, tension type headache $(T H)$, and hypothyroidism are clinical problems that affect patient daily activities and quality of life.

Objectives: The purpose of this study was to investigate the potential association between hypothyroidism in patients with migraine and TTH.

Patients and methods: Two hundred and twelve patients with migraine and $T \mathrm{TH}$ and one hundred control subjects underwent clinical evaluation, assessment of thyroid hormones, and thyroid ultrasound.

Results: Subclinical and overt hypothyroidism were significantly higher in patients with migraine and $T H \mathrm{H}(P=$ $0.001)$ than control subjects. Patients with migraine and TTH showed significantly more abnormal thyroid gland morphology than healthy control $(P=0.027)$. Hypothyroidism is significantly expressed in chronic $T H$ more than TTH with infrequent or frequent $T \mathrm{TH}(P=0.009)$.

Conclusions: Patients having migraine and TTH more prone to develop hypothyroidism when compared with control group. Also patients with chronic $\mathrm{TTH}$ are susceptible to develop hypothyroidism (either subclinical or overt) when compared with patients having frequent or infrequent $T \mathrm{H}$.
\end{abstract}

Keywords: Hypothyroidism, Tension type headache, Migraine, Thyroid hormones, Thyroid ultrasound

\section{Introduction}

Migraine and tension type headache (TTH) disorders are among the top six most prevalent disorders and the third cause of disability worldwide in individuals under the age of 50 with a major impact on activities of daily living and quality of life $[1,2]$.

Tension-type headache (TTH) is defined as a mild to moderate band-like pressure headache associated with some somatic and emotional symptoms. The frequency and duration of $\mathrm{TH}$ varied from infrequent episodes of a headache to frequent, long-lasting, or even chronic [3].

Typical characteristics of migraine headache are unilateral location, pulsating quality, moderate or severe intensity, aggravation by routine physical activity, and

\footnotetext{
* Correspondence: carmenali042@gmail.com

${ }^{1}$ Faculty of Medicine, Badr University Hospital, Helwan University, Cairo, Egypt Full list of author information is available at the end of the article
}

association with nausea and/or photophobia and phonophobia [4].

Hypothyroidism prevalence in general population in Europe ranged between 0.2 and $5.3 \%$ and usually this represents thyroid hormone deficiency. It affects women more than men and is more common in older age $(\geq 65$ years) with clinical burden related to major organs including neurological symptoms [5].

Hypothyroidism represents one of non-neurological comorbidities of migraine. These comorbidities (refers to the greater than coincidental association of separate conditions) significantly influence the progression of headache and may interact with choosing the therapeutic strategies $[6,2]$.

Recent studies explained the comorbidity by involvement of thyroid hormone in pain mechanisms with thyrotropin stimulating hormone receptors being expressed in the cortical neurons and cerebral vasculature [7]. 
Many studies defined a bidirectional relationship between migraine, $\mathrm{TH}$, and hypothyroidism including underlying pathophysiological aspects. However up till now, there is contradictory data about these relationship [8]. There is uncertainty in pathogenesis of development of migraine, tension $\mathrm{TH}$ in hypothyroidism; some proposed the alterations in the immune system and autonomic nervous system with shared genetic or environmental factors [9].

The purpose of this study was to investigate the potential association between hypothyroidism in patients with migraine and tension headache.

\section{Subjects and methods Subjects}

In a cross-sectional study, we investigated two hundred and twelve patients with migraine and tension type headache and one hundred, age- and sex-matched healthy control subjects. Patients were recruited from neurology outpatient clinics of Helwan University Hospitals, Egypt, from May 2019 to December 2019.

Inclusion criteria in this study were patients of both sex with age ranged from 18 to 55 years.

All patients underwent full history taking stressing on headache characteristics, severity, frequency, and medications used. Frequent headache attacks are defined by headache more than 10 attacks per month (at least 10 episodes of headache occurring on 1-14 days/month on average for $>3$ months ( $\geq 12$ and $<180$ days/year) $)$ [4]. Infrequent headache is defined as at least 10 episodes of headache occurring on $<1$ day/month on average $(<12$ days/year) $[4,10]$. Patients with migraine headache were diagnosed according to the International Classification of Headache Disorders (ICHD)-III beta criteria [8]. It includes recurrent headache manifesting in attacks lasting 4-72 h. Typical characteristics of the headache are unilateral location, pulsating quality, moderate or severe intensity, aggravation by routine physical activity, and association with nausea and/or photophobia and phonophobia. Chronic migraine is defined as headache occurring on 15 or more days per month for more than 3 months, which, on at least 8 days per month, has the features of migraine headache [4].

Patients with tension type headache was diagnosed according to the International Classification of Headache Disorders (ICHD)-III beta criteria [4]. All patients must have all of the following four headache characteristics: bilateral, mild-to-moderate intensity, non-pulsating headache not aggravated by routine physical activity, and their headaches must not be associated with any of nausea, vomiting, photophobia, or phonophobia. Chronic tension headache means that type of tension headache lasts hours and may be continuous and if headaches occur 15 or more days a month for at least 3 months [4,
11]. Normal neurological examination. Completely normal laboratory tests (complete blood count, creatinine, liver function tests)

Exclusion criteria included patients that were beyond the range of 18-55 years, showed abnormal neurological examination, and or had chronic illness known to affect thyroid dysfunction (chronic kidney disease, psychiatric disorders) were excluded from the study. Also, pregnant ladies and those with chronic drug intake that may alter thyroid hormone level as lithium carbonate and amoidarone were excluded.

\section{Methods}

Blood samples were collected. An enzyme-linked immunosorbent assay was performed to detect the basal serum concentrations of TSH, FT3, and FT4. This assay was processed according to the instructions and regulations of the used kits. Overt hypothyroidism was defined as elevated TSH $(>10 \mathrm{mU} / \mathrm{L})$ with low T4 $(\mathrm{T} 4<4.5 \mu \mathrm{g} /$ $\mathrm{dl})$, whereas subclinical hypothyroidism was defined as elevated TSH (TSH: > $4.5 \mathrm{mU} / \mathrm{L}$ ) with normal T4 (normal T4: 4.5-13 $\mu \mathrm{g} / \mathrm{dl}$ ) [12].

\section{Thyroid ultrasound}

All patients were examined in supine position with hyperextended neck, using a high frequency linear-array transducer 7-15 MHz (ultrasound machine; GE brand, Logiq. P5 pro model, GE health care manufacture, France). Scanning was done both in longitudinal and transverse planes. Real time imaging of thyroid lesions was performed using both grayscale and color Doppler techniques. Thyroid ultrasound examinations showed normal thyroid gland shape, size, and echo-pattern or abnormal thyroid ultrasound (diffuse increase size of both thyroid lobes with diffuse heterogeneous echopattern, diffuse increase size of both thyroid lobes with multiple nodules, or diffuse increase size of both thyroid lobes with normal echo-pattern).

For all the participants, written informed consent was obtained after the study procedures had been explained. The study protocol and the consent form were reviewed and accepted by the Research Ethic Committee (REC) for Human Subject Research at Faculty of Medicine, Helwan University, Cairo, Egypt (serial: 13-2019).

\section{Statistical analysis}

Data were entered and analyzed using the IBM-SPSS software (IBM Corp. Released 2017. IBM SPSS Statistics for Windows, Version 25.0. Armonk, NY: IBM Corp.). Qualitative data were expressed as frequency and percentage. Chi-square test or Fisher's exact test according to sample size of cells (expected counts) was used for comparing qualitative data. Chi-square test was used if sample size was $\geq 5$ units per cell while Fisher's exact 
test was used if sample size was $<5$ units in $\geq$ one cell. Quantitative data were initially tested for normality using Shapiro-Wilk's test with data being normally distributed if $p>0.050$. Presence of significant outliers (extreme values) was tested for by inspecting boxplots. Quantitative data were expressed as mean \pm standard deviation (SD) if normally distributed or median and interquartile range (IQR) if not. To compare quantitative data for two groups, independent-samples $t$ test was used for normally distributed data in both groups with no significant outliers otherwise the alternative nonparametric test (Mann-Whitney $U$ test) was used. To compare quantitative data for >two groups, one-way ANOVA test was used for normally distributed data in all groups with no significant outliers otherwise the alternative non-parametric test (Kruskal-Wallis $H$ test) was used. Binary logistic regression was used to predict the likelihood of occurrence of hypothyroidism. For any of the used tests, results were considered as statistically significant if $p$ value $\leq 0.050$. Appropriate charts were used to graphically present the results whenever needed [13].

\section{Results}

Our study included 212 patients (118 had tension type headache "TTH" and 94 had migraine headache) and 100 control subjects. There were no statistically significant differences between patients and control as regards age and sex distribution $(p=0.491$ and 0.449 respectively) Table 1.

Subclinical and overt hypothyroidism were significantly higher in our patients more than control $(p=$ 0.001 ). Moreover, our patients showed significantly more abnormal gland morphology than healthy control $(p=$ 0.027) Table 2.

Binary logistic regression (BLR) analysis was run to assess the influence of having migraine or TTH versus no headache on the likelihood that participants will exhibit hypothyroidism (subclinical or overt). The model was statistically significant $(p=0.001)$. The model correctly classifies $76.8 \%$ of cases. Patients with migraine or TTH had 3.73 times higher odds to exhibit hypothyroidism $(\mathrm{BLR}=1.316,95 \% \mathrm{CI}=1.82-7.64, p=0.001)$. Logistic regression analysis was also run to assess the influence of having chronic tension headache vs other types (frequent and infrequent) on the likelihood that participants will exhibit hypothyroidism (subclinical or overt). The model was statistically significant $(p=0.002)$. the model correctly classifies $71.2 \%$ of cases. Patients with chronic subtype had 3.57 times higher odds to exhibit hypothyroidism $(\mathrm{BLR}=1.272,95 \% \mathrm{CI}=1.55-8.22, p=$ 0.003) (Table 3).

Migraine patients were significantly younger than tension type headache patients $(p=0.001)$. There were no significant differences between both migraine and TTH as regards gender, thyroid function, or even abnormal thyroid morphology (Table 4).

Using intragroup comparative statistics, hypothyroidism is significantly expressed in chronic TTH more than TTH with infrequent or even frequent attacks $(p=0.009)$. Moreover, chronic TTH had older age than other TTH subgroups but not statistically significant differences $(p=$ 0.596). Thyroid gland abnormalities were more common in chronic TTH but with insignificant power $(p=0.227)$ (Table 5).

Intragroup analytical statistics of migraine patients regarding age of onset, disease duration, gender, and thyroid gland parameters were analyzed in Table 6 . There were no statistically significant differences between migraine with aura, migraine without aura, and chronic migraine as regards age, sex, thyroid functions, or thyroid abnormalities on ultrasound $(p>0.05)$.

\section{Discussion}

Tension-type headache and migraine are among the top six most prevalent diseases globally, and represent the third cause of disability worldwide in individuals under the age of 50 [14].

In pathogenesis of migraine attacks, the role of hormonal regulation is proved by the presence of its menstrual-dependent variants. But the data about the role of thyroid hormones in migraine not confirmed

Table 1 Age and sex distribution between cases and control

\begin{tabular}{llll}
\hline Characteristic & Case $(n=212)$ & Control $(n=100)$ & $p$ value \\
\hline $\begin{array}{l}\text { Age, mean (range) } \\
\text { Sex }\end{array}$ & $41(33-47)$ & $39(29-45)$ & \\
$\quad$ & $126(59.4 \%)$ & $55(55 \%)$ \\
Female: total no. (\%) & $37(17.5 \%)$ & $7(7 \%)$ \\
$\quad$ Female with hypothyroidism & $(13.7 \%$ and $3.7 \%)$ & $(6 \%$ and $1 \%)$ \\
$\quad$ Subclinical and overt) & $86(40.6 \%)$ & $45(45 \%)$ \\
Male: total no. (\%) & $26(12.3 \%)$ & $3(3 \%)$ \\
$\quad$ Male with hypothyroidism & $(10 \%$ and $2.3 \%)$ & $(3 \%$ and $0 \%)$ \\
$\quad$ Subclinical and overt) & &
\end{tabular}

$P$ value is significant if $\leq 0.050$ 
Table 2 Thyroid function and ultrasound between cases and control

\begin{tabular}{|c|c|c|c|}
\hline Characteristic & Case $(n=212)$ & Control $(n=100)$ & $p$ value \\
\hline Thyroid function & & & 0.001 \\
\hline Euthyroid: no. (\%) & $149(70.3 \%)$ & $90(90 \%)$ & \\
\hline Subclinical hypothyroidism: No. (\%) & $50(23.6 \%)$ & $9(9 \%)$ & \\
\hline Overt hypothyroidism: no. (\%) & $13(6.1 \%)$ & $1(1 \%)$ & \\
\hline Thyroid ultrasound & & & 0.027 \\
\hline Normal: no. (\%) & $190(89.6 \%)$ & 97 (97\%) & \\
\hline Abnormal: no. (\%) & $22(10.4 \%)$ & $3(3 \%)$ & \\
\hline
\end{tabular}

$P$ value is significant if $\leq 0.050$

with many contradictory studies [15]. Hagen and his colleagues found low headache prevalence among patients with hypothyroidism [16] while others observed high prevalence of chronic migraine in patients with hypothyroidism [17]. Migraine, tension headache, and hypothyroidism are clinical problems that affect patient daily activities and quality of live with established comorbidity that has been known for more than 60 years $[18,19]$.

Hypothyroidism in our study was more common in females than males (in both migraine and tension headache together) although not statistically significant ( $p=$ 0.448). The female preponderance in our study was consistent with Khan and his colleagues which found higher prevalence of both migraine and TTH in patients with hypothyroidism [20]. While other study reported that women with hypothyroidism were protected from headache [16].

We have found in our study of 212 patients (migraine and tension headache) that there was significantly higher proportion with subclinical (23.3\%) and overt hypothyroidism (6\%), as compared to the control subjects of $9 \%$ and $1 \%$. This was in agreement with the results from other studies [21, 22].

Lima Carvalho and his colleagues found in a population of 213 patients with de novo hypothyroidism (133 of them with subclinical hypothyroidism) that new onset headache developed in $34 \%$ of the patients, with a predominantly migraine phenotype, while $78 \%$ of patients

Table 3 Predictors of the likelihood of occurrence of hypothyroidism

\begin{tabular}{llll}
\hline Predictor & BLR & $p$ value & $95 \% \mathrm{Cl}$ \\
\hline Migraine and TTH & & & \\
$\quad$ No (control) & 1.316 & 0.001 & $\mathrm{R}$ \\
$\quad$ Yes (case) & & & 3.73 (1.82-7.64) \\
Tension headache & & & \\
$\quad$ Frequent, infrequent and chronic & 1.272 & 0.003 & $\mathrm{R}$ \\
& & & 3.57 (1.55-8.22) \\
\hline
\end{tabular}

$P$ value is significant if $\leq 0.050$

$\pi H$ tnsion type headache, $B L R$ binary logistic regression, $\mathrm{Cl}$ confidence interval (with both overt and subclinical hypothyroidism) treated with levothyroxine for 12 months demonstrated improvement of headache symptoms [23].

In contrast to our findings, a cross-sectional study of 130 out-patient migraineurs revealed abnormal levels of TSH only in $5 \%$ of patients, and also that lower TSH values were associated with more prolonged migraine attacks and with a greater impact on quality of life [24].

Moreover, our patients showed significantly more abnormal gland morphology than healthy control on thyroid gland ultrasound. The subclinical and overt hypothyroidism prevalence in our patients with migraine and tension headache were also higher than that reported in the general population. The prevalence of subclinical hypothyroidism in general population ranges from $4-10 \%$ [25] and that of overt hypothyroidism is 1$2 \%$ [26], and is in agreement with our control group.

In our study, binary logistic regression analysis showed that patients having migraine and TTH were more prone to develop hypothyroidism when compared with control group $(\mathrm{BLR}=1.316,95 \% \mathrm{CI}=1.82-7.64, p=$ 0.001). Also patients with chronic TTH are susceptible to develop hypothyroidism (either subclinical or overt) 3.57 times when compared with patients having frequent or infrequent TTH $(\mathrm{BLR}=1.272,95 \% \mathrm{CI}=1.55-8.22, p$ $=0.003)$.

There are several mechanisms that may explain the association between migraine and hypothyroidism. Changes in the immune system that occur as a result of migraine may predispose to thyroid autoimmunity [27].

In addition, migraineurs demonstrate sympathetic hypo function during interictal periods, which may increase the risk for hypothyroidism [28].

Genetic factors, such as hyperhomocystemia and shared environmental factors, including air pollutants and synthetic compounds [29] could also predispose to both migraine and hypothyroidism. Singh proposed a possible pathophysiological link between migraine and hypothyroidism. He suggested that pain is regulated by reciprocal modulation of brain stem serotonergic and noradrenergic nuclei. The failure of one of the two systems implies a compensatory response of the other. 
Table 4 Age, gender, and thyroid profile between tension headache and migraine

\begin{tabular}{|c|c|c|c|}
\hline Characteristic & Tension headache $(n=118)$ & Migraine $(n=94)$ & $p$ value \\
\hline Age, mean (range) & $43(37-48)$ & $36(28-44)$ & 0.001 \\
\hline Sex & & & 0.230 \\
\hline Female: no. (\%) & $67(56.8 \%)$ & $61(64.9 \%)$ & \\
\hline Male: no. (\%) & $51(43.2 \%)$ & $33(35.1 \%)$ & \\
\hline Thyroid function & & & 0.775 \\
\hline Euthyroid: no. (\%) & $84(71.2 \%)$ & $65(69.1 \%)$ & \\
\hline Subclinical hypothyroidism: no. (\%) & $28(23.7 \%)$ & $22(23.4 \%)$ & \\
\hline Overt hypothyroidism: no. (\%) & $6(5.1 \%)$ & $7(7.4 \%)$ & \\
\hline Thyroid ultrasound & & & 0.911 \\
\hline Normal: no. (\%) & $106(88.8 \%)$ & $84(89.4 \%)$ & \\
\hline Abnormal: no. (\%) & 12 (10.2\%) & 10 (10.6\%) & \\
\hline
\end{tabular}

$P$ value is significant if $\leq 0.050$

Thus, the decreased adrenergic tone in hypothyroidism could upregulate the serotonergic response which causes headache.

In our study, migraine patients were significantly younger than tension type headache patients $(p=0.001)$. This is in agreement with Okumura and his colleagues [30]. There were no significant differences between both migraine and TTH as regards gender, thyroid function or even abnormal thyroid morphology. About one-third of our patients showed abnormal thyroid function (29.7\%) whether subclinical or overt hypothyroidism and only $10.3 \%$ of them had abnormal gland morphology, these findings are consistent with many previous observation $[31,32]$.

In the study of Ekici and Cebeci, subclinical hypothyroidism was found in only (5.1\%) of patients with migraine [33] while Parashar and his colleagues did not find any changes in thyroid hormone levels with migraine or TTH [34], Larner did not reveal migraine headache associated with hypothyroidism [35], and hypothyroidism were associated with lower frequency of occurrence of headaches in all age groups [16].

In our study, the frequency of tension headache was $55.7 \%$ and migraine headache $44.3 \%$ and this observation is partially agreed with Hagen and his colleagues [36] as they reported that prevalence of TTH was $51.9 \%$, meanwhile for migraine was $17.2 \%$. Moreover, Stovner and his colleagues found $11 \%$ of their patients had migraine and $42 \%$ had tension-type headache [37].

Intragroup comparative analysis of TTH patients revealed that patients with chronic TTH were significantly older than other subtypes $(p=0.010)$ and have longer disease duration than episodic TTH $(p=0.001)$. Moreover, $46.3 \%$ of those patients suffered from chronic TTH have overt hypothyroidism versus $20 \%$ and $19.1 \%$ of those with frequent TTH and infrequent TTH respectively $(p=0.009)$, this is in agreement with Khan and his colleagues reported that frequency of hypothyroidism

Table $\mathbf{5}$ Intragroup analysis between tension headache subtypes

\begin{tabular}{|c|c|c|c|c|}
\hline Characteristic & Frequent TTH $(n=30)$ & Infrequent TTH $(n=47)$ & Chronic TTH $(n=41)$ & p \\
\hline Age (years): mean $\pm S D$ & $39.3 \pm 7.1$ & $42.3 \pm 7.3$ & $44.5 \pm 6.3 b$ & 0.010 \\
\hline Duration (years) median (range) & $6.5(3.8-11)$ & $10(5-14)$ & $13(11-16)$ & 0.001 \\
\hline Sex & & & & 0.596 \\
\hline Female: no. (\%) & $15(50 \%)$ & $29(61.7 \%)$ & $23(56.1 \%)$ & \\
\hline Male: no. (\%) & $15(50 \%)$ & $18(38.3 \%)$ & $18(43.9 \%)$ & \\
\hline Thyroid function & & & & 0.009 \\
\hline Euthyroid: no. (\%) & $24(80 \%)$ & 38 (80.9\%) & $22(53.7 \%)$ & \\
\hline Hypothyroidism: no. (\%) & $6(20 \%)$ & $9(19.1 \%)$ & $19(46.3 \%)$ & \\
\hline Thyroid ultrasound & & & & 0.227 \\
\hline Normal: no. (\%) & $28(93.3 \%)$ & $44(93.6 \%)$ & $34(82.9 \%)$ & \\
\hline Abnormal: no. (\%) & $2(6.7 \%)$ & $3(6.4 \%)$ & $7(17.1 \%)$ & \\
\hline
\end{tabular}

$P$ value is significant if $\leq 0.050$

$\pi \mathrm{T}$ tension type headache 
Table 6 Intragroup analysis between migraine subtypes

\begin{tabular}{|c|c|c|c|c|}
\hline Characteristic & With aura $(n=9)$ & Without aura $(n=53)$ & Chronic $(n=32)$ & $p$ \\
\hline Age (years): mean \pm SD & $36.4 \pm 12.3$ & $34.7 \pm 9.2$ & $37.9 \pm 9.6$ & 0.322 \\
\hline Duration (years) median (range) & $14(7-18.5)$ & $10(6-13)$ & $10(5.3-15)$ & 0.400 \\
\hline Sex & & & & 0.586 \\
\hline Female: no. (\%) & $6(66.7 \%)$ & $32(60.4 \%)$ & $23(71.9 \%)$ & \\
\hline Male: no. (\%) & $3(33.3 \%)$ & $21(39.6 \%)$ & $9(28.1 \%)$ & \\
\hline Thyroid function & & & & 0.137 \\
\hline Euthyroid: no. (\%) & $5(55.6 \%)$ & $41(77.4 \%)$ & 19 (59.4\%) & \\
\hline Hypothyroidism: no. (\%) & $4(44.4 \%)$ & $12(22.6 \%)$ & $13(40.6 \%)$ & \\
\hline Thyroid ultrasound & & & & 0.468 \\
\hline Normal: no. (\%) & $8(88.9 \%)$ & $49(92.5 \%)$ & 27 (84.4\%) & \\
\hline Abnormal: no. (\%) & $1(11.1 \%)$ & $4(7.5 \%)$ & $5(15.6 \%)$ & \\
\hline
\end{tabular}

$P$ value is significant if $\leq 0.050$

was more common in chronic TTH as compared to episodic TTH [38]. More recently, Qu with his colleagues [39] observed in chronic TTH there were lower levels of T3 and T4 and higher level of TSH than controls.

Regarding sub analysis of migraine patients, there were no significant differences between migraine with aura, migraine without aura, or chronic migraine as regards thyroid function or morphology $(p=0.137$ and $p=$ 0.468 respectively) and this is consistent with a recent Russian study who reported negative results regarding comorbidities of migraine and hypothyroidism with abnormal levels of TSH in only $5 \%$ of their migraine patients. On the other hand, many other studies [40, 41] found that hypothyroidism was statistically significantly higher in chronic migraine when compared with migraine without aura but when compared with migraine with aura it was statistically insignificant.

\section{Conclusion}

Hypothyroidism is a common comorbidity with migraine and TTH. Patients having migraine and TTH more prone to develop hypothyroidism when compared with control group. Also patients with chronic TTH are susceptible to develop hypothyroidism (either subclinical or overt) when compared with patients having frequent or infrequent TTH.

\section{Limitations of the study}

We did not investigate the etiopathological relationship between hypothyroidism and other types of headache disorders whether there is a true interactions or just by a chance. We did not address the effect of treatment of thyroid dysfunction on severity and the course of headache. Moreover, the limited number of patients and control (small sample size) beside we did not recruit other cases of primary headache disorder may be a limitation of our study.

\section{Recommendations}

Study the response of patients suffering from migraine or tension headache using conventional measures and having hypothyroidism when controlling thyroid function. Sufficient number of patients with migraine, tension headache and control subjects for detection the accurate relationship. Study the relationship between other types of primary headache disorders and hypothyroidism. Moreover, study if there is relationship between primary headache disorders and thyroid dysfunction whether hypo or hyperthyroidism.

\section{Abbreviations}

FET: Fisher's exact test; ICHD-III: International Classification of Headache Disorder version III; IQR: Interquartile range; K.W: Kruskal-Wallis; REC: Research Ethical Committee; SD: Standard deviation; TTH: Tension type headache; TSH: Thyroid stimulating hormone; T4: Thyroxine; T3: Triiodothyronine; Us: Ultrasound

\section{Acknowledgements \\ The authors are grateful to all patients and control subjects for their willingness to participate in this study.}

\section{Authors' contributions}

AAM, MEF, TB, and CAZ carried out the work. AAM designed the study and collected the patients and gathered the clinical data. MEF did the statistical analysis. TB was responsible for collecting the scientific data and writing the initial draft of the manuscript. CAZ collected and reported the radiological data of all patients US. The authors read and approved the final version to be published.

Funding

There is no source of funding for the research.

\section{Availability of data and materials}

The data supporting the results of this article are included within the article.

\section{Ethics approval and consent to participate}

The authors obtained permission to conduct this study and was approved by Research Ethic Committee (REC) for Human Subject Research at Faculty of Medicine, Helwan University, Cairo, Egypt (serial: 13-2019) and all participants gave written informed consent. The procedures followed were in accordance with our protocol. An informed consent was obtained from all the patients studied. 


\section{Consent for publication}

Not applicable.

\section{Competing interests}

The authors declare that they have no competing interests.

\section{Author details}

${ }^{1}$ Faculty of Medicine, Badr University Hospital, Helwan University, Cairo, Egypt. ${ }^{2}$ Faculty of Medicine, Mansoura University, Mansoura, Egypt. ${ }^{3}$ Faculty of Medicine, Port Said University, Port Said, Egypt.

\section{Received: 1 April 2020 Accepted: 1 July 2020}

\section{Published online: 16 July 2020}

\section{References}

1. Vos T, Abajobir AA, Abate KH, Kaja A, Abbas M, Agrawa A, et al. Global, regional, and national incidence, prevalence, and years lived with disability for 328 diseases and injuries for 195 countries, 1990-2016: a systematic analysis for the global burden of disease study 2016. Lancet. 2017;390:121159.

2. Steiner TJ, Stovner LJ, Vos T. GBD: migraine is the third cause of disability in under 50s. J Headache Pain. 2016;17:104.

3. Headache Classification Committee of the International Headache S. The International Classification of Headache Disorders, 3rd edition (beta version). Cephalalgia. 2013;33(9):629-808.

4. Headache Classification Committee of the International Headache Society (IHS) The International Classification of Headache Disorders, 3rd edition. Cephalalgia. 2018 Jan;38(1):1-211.

5. Spanou I, Bougea A, Liakakis G, Rizonaki K, Anagnostou E, Duntas L, et al. Relationship of migraine and tension-type headache with hypothyroidism: a literature review. Headache. 2019;59(8):1174-86.

6. Feinstein AR. The pre-therapeutic classification of co-morbidity in chronic disease. J Chronic Dis. 1970;23:455-68.

7. Aloisi AM, Vodo S, Buonocore M. Pain and thyroid hormones. Neurol Sci. 2013;34:1501-8.

8. Lisotto C, Mainardi F, Maggioni F, Zanchin G. The comorbidity between migraine and hypothyroidism. J Headache Pain. 2013;1:138.

9. Rainero I, Govone F, Gai A, Vacca A, Rubino E. Is migraine primarily a metabolon-endocrine disorder? Curr Pain Headache Rep. 2018;22:36

10. Schwedt TJ. Chronic migraine. BMJ. 2014;348:1416.

11. Bendtsen $L$, Evers $S$, Linde $M$, et al. EFNS guideline on the treatment of tension-type headache - report of an EFNS task force. Eur J Neurol. 2010;17: 1318.

12. Fatourechi V. Subclinical hypothyroidism: an update for primary care physicians. Mayo Clin Proc. 2009;84(1):65-71.

13. Heyen Petra. The principle of biomedical statistics: Masur H, Papke K, Althoff S., andOberwittler C.: Scales and scores in neurology; quantification of neurologicaldeficit in research and practice. Thieme Stuttgart New York 2004; 3: 13-51

14. Leonardi M, Raggi A. Burden of migraine: international perspectives. Neurol Sci. 2013;34(Suppl 1):S117-8.

15. Starikova NL, Baidina TV, Kalashnikova TP. Thyrotropin levels and severity of symptoms in migraine patients of tertiary headache center. International Headache Society. 2018:1-5.

16. Hagen $K$, Bjero T, Zwart JA, Vatten L, Stovner LJ, Bovim G. Low headache prevalence amongst women with high TSH values. Eur J Neurol. 2001;8: 693-9.

17. Bigal ME, Sheftell FD, Rapoport AM, Tepper SJ, Lipton RB. Chronic daily headache: identification of factors associated with induction and transformation. Headache. 2002:42:575-81.

18. Younger DS. Epidemiology of migraine. Neurol Clin. 2016;34(4):849-61.

19. Fallah R, Mirouliaei M, Bashardoost N, Partovee M. Frequency of subclinical hypothyroidism in 5-15 years old children with migraine headache. J Pediatr Endocrinol Metab. 2012;25:859-62.

20. Khan HB, Shah PA, Bhat MH, Imran A. Association of hypothyroidism in patients with migraine and tension type headache disorders in Kashmir. North India. Neurology Asia. 2015;20(3):257-26.

21. Olesen J. The International Classification of Headache Disorders, 2nd Edition. Cephalgia. 2006;26(12):1409-10.

22. Singh SK. Prevalence of migraine in hypothyroidism. J Assoc of Phys of India. 2002;50:1455-6.
23. Lima Carvalho MF, de Medeiros JS, Valenca MM. Headache in recent onset hypothyroidism: prevalence, characteristics and outcome after treatment with levothyroxine. Cephalalgia. 2017;37:938-46.

24. Starikova NL, Baidina TV, Kalashnikova TP. Thyrotropin levels and severity of symptoms in migraine patients of tertiary headache center. Cephalalgia. 2019:39:148-52.

25. Gharib H, Tuttle RM, Baskin HJ, Fish LH, Peter A. Singer PA, et al. Subclinical thyroid dysfunction: a joint statement on management from the American Association of Clinical Endocrinologists, the American Thyroid Association, and the Endocrine Society. Endocrine Practice 2005; 10:1:581-585.

26. Tietjen GE, Herial NA, Hardgrove J, Utley C, White L. Migraine comorbidity constellations. Headache. 2007;47(6):857-65.

27. Waeber C, Moskowitz MA. Migraine as an inflammatory disorder. Neurology. 2005;64:S9-S15.

28. Silva JE, Bianco SD. Thyroid-adrenergic interactions: physiological and clinical implications. Thyroid. 2008;18:157-65.

29. Szyszkowicz M, Kaplan GG, Grafstein E, Rowe BH. Emergency department visits for migraine and headache: a multi-city study. Int J Occup Med Environ Health. 2009;22:235-42.

30. Robles-Osorio ML, Zacarias-Rangel V, GarclaSolis P, Hernandez-Montiel HL, Solis JC, et al. Prevalence of thyroid function test abnormalities and antithyroid antibodies an open population in central Mexico. Rev Investig Clin. 2014;66:113-20.

31. Amy JR. Tests of thyroid function in chronic headache patients. Headache. 1987;27:351-3.

32. Ekici B, Cebeci AN. The debate on the link between subclinical hypothyroidism and childhood migraine: is initial endocrinological evaluation necessary for children with migraine? Acta Neurol Belg. 2015;115: 123-7.

33. Parashar R, Bhalla P, Rai NK, Pakhare A, Babba R. Migraine: is it related to hormonal disturbances or stress? Int J Womens Health. 2014;6:921-5.

34. Larner AJ. Thyroid dysfunction and headache. J Headache Pain. 2006;7:51-2.

35. Okumura T, Tanno S, Ohhira M, Tanno S, Nozu T. Characteristics in patients with headache in an outpatient clinic in Japan. Asia Pacific Family Medicine. 2010;9:10

36. Rubino E, Rainero I, Garino F, Vicentini C, Govone F, Vacca A, et al. Subclinical hypothyroidism is associated with migraine: a case-control study. Cephalalgia. 2019;39:15-20

37. Stovner LJ, Al Jumah M, Birbeck GL, Gururaj G, Jensen R, Katsarava Z, et al. The methodology of population surveys of headache prevalence, burden and cost: principles and recommendations from the Global Campaign against Headache. J Headache Pain. 2014;27:15-5.

38. Hagen $K$, Zwart JA, Aamodt AH, Nilsen KB, Bråthen G, Helde G. etal. A faceto-face interview of participants in HUNT 3: the impact of the screening question on headache prevalence. J Headache Pain. 2008;9:289-94.

39. Qu P, Yu JX, Xia L, Chen GH. Cognitive performance and the alteration of neuroendocrine hormones in chronic tension-type headache. Pain Pract. 2018:18:8-17.

40. Le H, Tfelt-Hansen P, Russell MB, Skytthe A, Kyvik KO, Olesen J. Co-morbidity of migraine with somatic disease in a large population-based study. Cephalalgia. 2011;31:43-64

41. Sheftell ME, Rapoport FD, Alan M, Tepper AM, Stewart J, Richard B. Chronic daily headache: identification of factors associated with induction and transformation. Headache. 2002;42(7):575-81.

\section{Publisher's Note}

Springer Nature remains neutral with regard to jurisdictional claims in published maps and institutional affiliations. 